\title{
Efecto de mallas raschel de colores en el crecimiento de plántulas de Eucalyptus globulus, Eucalyptus nitens y Pinus radiata en condiciones de vivero
}

\author{
Colored raschel mesh effect on growth of Eucalyptus globulus, \\ Eucalyptus nitens and Pinus radiata seedlings under nursery conditions
}

\author{
Angela Bustos-Salazar a, Alejandra Zuñiga-Feest ${ }^{\text {b* }}$ \\ ${ }^{a}$ Universidad Austral de Chile, Facultad de Ciencias Forestales y Recursos Naturales, \\ Centro de Ciencia del Clima y Resiliencia $\left(\mathrm{CR}^{2}\right)$, Escuela de Graduados, Valdivia, Chile. \\ *Autor de correspondencia: ${ }^{\mathrm{b}}$ Universidad Austral de Chile, Facultad de Ciencias, Instituto de Ciencias Ambientales y Evolutivas, \\ Laboratorio de Biología Vegetal, Valdivia, Chile, alejandrazunigafeest@gmail.com
}

\begin{abstract}
SUMMARY
Forest plantations in Chile represent 2.5 million hectares, with Eucalyptus spp. and Pinus as the most commonly used species. Worldwide, raschel meshes have been used in fruit, horticultural and ornamental shrubs to improve the production of fruits, leaves and flowers. In Chile, it has been operationally used for nursery protocols; they include raschel color mesh to increase shade, which leads to shoot elongation and protection against freezing. However, the possible effect of these colored meshes on the physiological performance of these seedlings is unknown. The objective of this work was to describe the light environment produced by these colored meshes (black, white, blue, green) and to evaluate their effect on growth and physiological performance of Pinus radiata, Eucalyptus nitens and E. globulus seedlings. To evaluate differences among colored meshes, the quality and intensity of light was determined. Seedlings maintained under each mesh were evaluated through the measurement of growth (increasing height of shoots), biomass distribution and vitality (fluorescence of PSII, Fv/Fm). Results show that black and white mesh influences were not selective on their transmittance spectrum and only provided shade (70\% and $50 \%$, respectively). Vitality measurements did not show damage in seedlings. Seasonal variation on growth was observed, with the highest values during fall and spring for P. radiata and only in spring for both Eucalyptus spp. White mesh promotes higher growth only on both Eucalyptus species, thus it would be recommended for nursery practices.
\end{abstract}

Key words: light quality, growth rate, fluorescence of PSII, Fv/Fm, raschel colored mesh, forestry nurseries.

\section{RESUMEN}

En Chile, las plantaciones forestales suman alrededor de 2,5 millones de hectáreas, siendo Pinus radiata y Eucalyptus spp. las especies más frecuentes. A nivel mundial, las mallas raschel se aplican en especies frutales, hortícolas y arbustos ornamentales para mejorar la producción de frutos, hojas y flores. En Chile, se han utilizado mallas raschel en la producción de plantas en viveros forestales para otorgar sombra y protección contra heladas. Sin embargo, se desconocen posibles efectos a nivel fisiológico en estas plantas. El objetivo de este trabajo fue caracterizar el ambiente lumínico generado por dichas mallas (negra, blanca, azul, verde) y evaluar el posible efecto sobre el crecimiento y el desempeño fisiológico de plántulas de Pinus radiata, Eucalyptus nitens y E. globulus. Para evaluar posibles diferencias en la calidad y cantidad de luz bajo cada malla de color, se determinó la transmitancia y la intensidad lumínica. A nivel de plantas, se evaluó el crecimiento en altura, distribución de biomasa y vitalidad (fluorescencia del fotosistema II, $\mathrm{Fv} / \mathrm{Fm}$ ). Los principales resultados indicaron que las mallas raschel negra y blanca no fueron selectivas en su espectro de transmisión y sólo aportaron sombra (70 \% y 50 \%, respectivamente). No se observaron daños en la vitalidad (Fv/Fm) en los tratamientos. Se observó un marcado crecimiento estacional; con máximos en otoño y primavera para $P$. radiata y solo en primavera, para ambas especies de Eucalyptus. Solo en las dos especies de Eucalyptus la malla blanca promovió mayor crecimiento, siendo la más recomendable para viveros forestales.

Palabras clave: calidad lumínica, tasa de crecimiento, fluorescencia del PSII, Fv/Fm, mallas raschel de color, viveros forestales.

\section{INTRODUCCIÓN}

Las plantaciones forestales en Chile representan cerca de 2,5 millones de hectáreas que corresponden, en su mayoría a Pinus radiata D. Don, Eucalyptus globulus La- bill, y Eucalyptus nitens Deane et Maiden con un 60, 23 y $10 \%$ de participación, respectivamente (INFOR 2015). Las plantas que abastecen esta demanda son generadas en vivero, mediante técnicas de mejoramiento genético a través de cruzamientos controlados y selección de proceden- 
cias. Uno de los problemas que enfrenta la producción de plantas en viveros forestales, es aumentar la tasa de crecimiento, sin disminuir los requerimientos de calidad, tales como: altura mínima, relación vástago/raíz, distribución homogénea de raíces, biomasa total y diámetro de cuello (DAC) mínimo (Haase 2008, Quiroz y Gutiérrez 2014). Es frecuente que un porcentaje aproximado de $30 \%$ de plantas de vivero, no cumplan con los requerimientos de altura y DAC, impidiendo de esta forma su despacho para plantaciones $^{1}$. Otro factor que afecta significativamente el tamaño final de las plantas forestales es el tipo y tamaño del contenedor. Se ha reportado que, tanto la tasa de crecimiento como el crecimiento estacional aumentan con el volumen del contenedor, esto también ha sido observado en otras especies producidas en viveros forestales como Pinus contorta Douglas ex Loudon y Picea glanca (Moench) Voss (Landis 1990). Adicionalmente, el volumen del contenedor influencia la relación tallo/raíz, la cual es crítica en el establecimiento de las especies forestales, especialmente en sitios con restricciones de humedad (Landis 1990).

El clima es uno de los factores que determina el nivel o ritmo de producción anual en plantaciones forestales y a nivel más local, el suelo es otro factor decisivo en el establecimiento de estas (Schlatter et al. 2003). Las plantaciones forestales se establecen en sitios sin cobertura vegetal, producto principalmente del manejo de desechos y control de malezas aplicado previo a su establecimiento. Por ende, estos sitios se encuentran expuestos a alta intensidad lumínica, requiriéndose que dichas plantas tengan un estándar de calidad suficiente para asegurar su sobrevivencia.

En viveros forestales el uso de mallas raschel de color negro para sombrear las platabandas e invernaderos, ha sido ampliamente utilizado desconociéndose cómo modifican el ambiente lumínico que reciben dichas plantas. Se ha observado que el uso de malla raschel negra promueve la elongación, generando plantas suculentas y poco lignificadas. Posteriormente, para el endurecimiento de las plantas se acostumbra a retirar las mallas al comienzo del verano, en forma progresiva, incrementando desde unas horas cada día, hasta retirarlas en forma definitiva antes de ser despachadas en la temporada otoño-invierno para la plantación. Es ampliamente conocido que, tanto a nivel morfológico como fisiológico, el ambiente lumínico (calidad, cantidad de luz y fotoperiodo) induce respuestas en las plantas, que involucran variación en: la concentración de pigmentos (ej. clorofila, xantofilas), nitrógeno foliar, tasa de crecimiento, distribución de biomasa, grosor de las hojas y ordenamiento de cloroplastos a nivel celular, entre otras respuestas (Terashima et al. 2005, Lambers et al. 2008). El crecimiento de las plantas y su desarrollo, son particularmente sensibles a cambios en el ambiente lumínico y especialmente a la sombra causada por plantas vecinas. Estas respuestas están controladas principal-

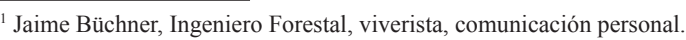

mente por fitocromos, que son moléculas receptoras de la relación R/RL (luz roja/luz roja lejana). Las plantas son capaces de responder ante la disminución de radiación fotosintéticamente activa (R), cuando es interceptada por plantas vecinas y se reduce la relación R/RL. En plantas que requieren alta intensidad lumínica, como son la mayor parte de las especies forestales que crecen en vivero, cuando la sombra aumenta y se reduce el valor de R/RL, ocurre una reasignación de recursos en la planta para favorecer la elongación del tallo y así alcanzar la luz del sol. La calidad de luz o valor de R/RL también se relaciona con la inducción en la germinación de las semillas y la anatomía foliar, en especial, el incremento de área de las hojas cuando su valor disminuye (Lorrain et al. 2008). Respuestas a nivel, fisiológico, morfológico y bioquímico por parte de las plantas permiten que éstas puedan hacer frente a factores de estrés abiótico, como es la sombra extrema (Myers y Kitajima 2007).

Para modificar el crecimiento, estructura y/o arquitectura de las plantas, principalmente en ornamentales y frutales se ha utilizado de manera exploratoria las mallas raschel de colores desde hace varios años, sin conocerse sistemáticamente sus efectos a nivel morfológico y fisiológico. Las mallas de colores deberían modificar la calidad y cantidad de luz disponible para las plantas, sin embargo, son escasos los ensayos que reporten estos posibles efectos (Oren-Shamir et al. 2001). Los trabajos existentes sobre el efecto de la calidad lumínica en plantas han utilizado principalmente filtros de papel de distintos colores (Folta 2004, Lazo y Ascencio 2010). En tanto, algunos trabajos con mallas raschel de colores han evaluado su efecto sobre fructificación, crecimiento y arquitectura de arbustos como Vaccinium myrtillus L. (arándano) (Rodríguez y Morales 2015) y Pittosporum variegatum (arbusto perenne, ornamental) (Oren - Shamir et al. 2001). Sin embargo, se desconoce el efecto que podrían tener sobre especies forestales de alta demanda. Los proveedores de estas mallas señalan que se obtiene un $65 \%$ de sombreamiento con su uso, pero se desconoce el efecto de estas sobre la "calidad lumínica". Se hipotetiza que la calidad e intensidad lumínica entregada por las mallas de colores difiere entre ellas, las que por tanto promoverán distintas respuestas en la tasa de crecimiento, distribución de biomasa y/o vitalidad de las plantas. El objetivo de este trabajo es caracterizar la calidad y cantidad lumínica entregada a las plantas bajo mallas raschel color negro, blanco, azul y verde, en adición a un control sin malla para evaluar respuestas morfológicas (altura, biomasa total, razón vástago/raíz y área foliar específica) y fisiológicas (fluorescencia de la clorofila del PSII, usando el parámetro $\mathrm{Fv} / \mathrm{Fm}$, como indicador de vitalidad) en plantas de Eucalyptus globulus, E. nitens y $P$. radiata, en condiciones de vivero. Se estudia también la interacción entre el efecto de las mallas de distintos colores y las posibles limitaciones causadas por el tamaño del contenedor. 


\section{MÉTODOS}

Área de estudio. El ensayo se realizó en la ciudad de Valdivia $\left(39^{\circ} 48^{\prime} \mathrm{S}\right.$ y $\left.73^{\circ} 14^{\prime} \mathrm{O}\right)$ a $19 \mathrm{~m}$ s.n.m. El clima es templado lluvioso de costa occidental con una precipitación anual de $2.540 \mathrm{~mm}$ y temperatura media anual de $11,9^{\circ} \mathrm{C}$. Valdivia se encuentra en un área con clima templado, con una influencia específica de un bioclima templado sub hiper oceánico, con baja oscilación térmica (Luebert y Pliscoff 2006).

Origen del material vegetal. Las plántulas de Pinus radiata fueron producidas usando estacas, (propagación vegetativa por estacas leñosas de familias, mejoradas mediante cruzamientos controlados) y las plántulas de Eucalyptus nitens y E. globulus fueron producidas a partir de semillas, desde árboles donde se realizaron cruzamientos controlados.

Mediciones de calidad e intensidad luminica (Steubing et al. 2002). Para determinar el efecto de la calidad e intensidad lumínica, se trabajó con mallas raschel de $65 \%$ de cobertura (entramado) en color blanco, negro, azul y verde. Para determinar el efecto selectivo del espectro de calidad de luz transmitida por las mallas raschel, se realizó un barrido de absorbancia (A) a distintas longitudes de onda, desde los 350 - 700 nanómetros usando un espectrofotómetro UV-VIS (Thermo, Helios gama, USA). Para ello se cortó un trozo de cada malla raschel y se puso en una cubeta adaptada para montar en el espectrofotómetro. Para calcular la transmitancia relativa (T) se utilizó la siguiente fórmula [1]:

Transmitancia relativa $=1 / 10 \times$ Absorbancia relativa

Para determinar el porcentaje de sombra de cada malla (atenuación de la intensidad lumínica), se registraron cinco mediciones instantáneas de la intensidad lumínica usando un radiómetro (LI $191 \mathrm{~S}$, Licor Inc., Lincoln NE, USA) en tres horas del día (8:00, 12:00 y 18:00), registrando la intensidad lumínica a plena luz y bajo cada malla raschel de color. El radiómetro utilizado posee varios sensores de luz que integran tanto la luz directa incidente, como la difusa que normalmente es reflejada en los ambientes. Se calculó el porcentaje de atenuación usando la siguiente fórmula [2]:

Porcentaje de atenuación $=$ (IL bajo malla/IL a plena luz) x 100

IL: intensidad lumínica en $\mu \mathrm{mol}$ de fotones $\mathrm{m}^{-2} \mathrm{~s}^{-1}$.

Diseño experimental. El diseño correspondió a bloques al azar. Los tratamientos correspondieron a mallas raschel de distintos colores (negro, azul, verde y blanco) además de un tratamiento sin malla. Para ello se realizaron case- tas con un armazón de madera de 70 × 70 x $130 \mathrm{~cm}$, las cuales fueron cubiertas completamente por cada tipo de malla raschel. El total de plantas en el ensayo fueron 70 para Pinus radiata (14 plantas por tratamiento), 50 para Eucalyptus nitens (10 plantas por tratamiento) y 50 para Eucalyptus globulus (10 plantas por tratamiento). Para estudiar la posible interacción entre el efecto de las mallas de distintos colores y las posibles limitaciones causadas por el tamaño del contenedor, después de nueve meses la mitad de las plántulas fueron trasplantadas desde un contenedor de $130 \mathrm{~cm}^{3}$ a uno de $500 \mathrm{~cm}^{3}$. Además, se consideraron plantas totalmente sanas para el ensayo, con un estado fitosanitario y vitalidad adecuados (con un valor de $\mathrm{Fv} / \mathrm{Fm} \geq 0,8)$. El sustrato que se utilizó para la producción de plantas en vivero correspondió a una mezcla de corteza compostada de pino con fertilizante superfosfato triple (6 $\left.\mathrm{mg} \mathrm{kg}{ }^{-1}\right)$, y fungicida postanol $\left(0,32 \mathrm{mg} \mathrm{kg}^{-1}\right)$.

Mediciones de respuestas morfológicas. Se midió la altura $(\mathrm{cm})$ de todas las plantas desde marzo a noviembre en intervalos de tres meses, para determinar incrementos relativos en altura. Estos incrementos se calcularon entre distintos periodos: a) marzo-mayo, b) mayo-julio, c) julioseptiembre y d) septiembre-noviembre. Cada cálculo de crecimiento fue relativo al último mes del periodo anterior según la fórmula [3]. El 5 de septiembre se trasplantó la mitad de las plantas a contenedor de $500 \mathrm{~cm}^{3}$ para evaluar el efecto de un mayor volumen disponible para raíces en el crecimiento. Finalmente, se cosecharon cuatro plantas de E. globulus, cuatro de E. nitens y seis plantas de $P$. radiata por tratamiento y por volumen de contenedor, separando entre raíz, tallo y hojas (70 plantas en total) para obtener la distribución de biomasa relativa por cada parte de la planta. Las muestras de tejido fueron secadas en estufa (Venticell; modelo: MMM Medcenter Einrichtunga Gmbh, Alemania) a $60{ }^{\circ} \mathrm{C}$ por tres días $(72 \mathrm{~h}$ ), hasta alcanzar un peso constante y pesadas utilizando una balanza analítica (RADWAG. AS / C 220 / C / 2 Poland). Con estos datos se determinó la distribución de biomasa, biomasa total (suma de todas las partes de la planta seca) y relación vástago/raíz.

$$
\begin{gathered}
\text { Incremento relativo en altura }= \\
((\mathrm{H} \text { final- } \mathrm{H} \text { inicial }) / \mathrm{H} \text { inicial }) \times 100
\end{gathered}
$$

Donde, H: altura (cm).

En el momento de la cosecha final en ambas especies de Eucalyptus, se separó el tercer par de hojas contadas desde el ápice y expandidas completamente en cada planta. Cabe mencionar que se marcó con pintura blanca un sector pequeño del último par de hojas expandidas, previo al ensayo con las mallas raschel de colores, por lo tanto, las hojas colectadas habían sido formadas durante el tratamiento con las distintas mallas (tercer par de hojas). Posteriormente, las hojas fueron colectadas y el área total 
se midió con un areafoliómetro (LI 3100, Licor Inc., Lincoln NE, USA). Para comparar la anatomía foliar de las hojas de Eucalyptus spp., formadas bajo las distintas mallas raschel de colores, se calculó el área foliar específica (specific leaf area, SLA) según fórmula [4]. Para obtener el peso seco, se secaron las hojas en estufa hasta llegar a peso constante durante $72 \mathrm{~h}$, posteriormente se pesó en balanza analítica, como fue descrito previamente. El área foliar específica no fue evaluada en Pinus radiata, debido a que sus hojas son aciculares, las cuales no presentan adecuaciones de SLA, comparado con Angiospermas ( $E u$ calyptus spp.) (Reich et al. 1997).

Área foliar específica $=$ Superficie foliar $\left(\mathrm{cm}^{2}\right) /$ Peso seco (g)

Medición del desempeño del fotosistema II. Parámetros tales como la eficiencia cuántica máxima $(\mathrm{Fv} / \mathrm{Fm}$; fluorescencia variable/ fluorescencia máxima) indican la eficiencia fotoquímica del fotosistema II (PSII) y es usado como un indicador de vitalidad de las plantas, bajo los distintos tratamientos. Este parámetro se midió usando un fluorímetro modulado (Hansatech modelo FMS2 Inglaterra). Los valores de $\mathrm{Fv} / \mathrm{Fm}$ considerados dentro de un rango óptimo son de $\approx 0,83$, siendo ampliamente usado en evaluaciones de estrés en plantas (Baker 2008). Las mediciones se realizaron en dos momentos del ensayo: invierno y primavera. Se utilizaron seis plántulas por cada tratamiento en cada especie, en la cual se midió en una hoja (una medición por planta). Para ello se seleccionaron las hojas totalmente expandidas bajo mallas raschel de color y en el tratamiento sin malla, ubicadas en el tercio superior de la planta. Las hojas fueron oscurecidas por 30 minutos con una pinza ad hoc y posteriormente se aplicó un pulso de luz saturante en esta misma zona de la hoja para obtener la fluorescencia máxima, siguiendo la metodología descrita en Bustos-Salazar (2011).

Análisis estadístico. Se realizó análisis de varianza (ANDEVA) de una y dos vías, para evaluar el efecto de las mallas raschel de colores (un factor) pudiendo establecer diferencias significativas entre tratamientos, y el posible efecto del volumen de contenedor (segundo factor) y la interacción entre ambos factores, en algunos parámetros y respuestas tanto a nivel morfológico como fisiológico. Entre ellos se encuentran: área foliar específica, relación vástago/raíz, biomasa total, distribución de biomasa y crecimiento relativo en altura total y por cada periodo de evaluación. Una vez establecidas las diferencias, se realizó una prueba de Tukey a posteriori para determinar los valores que presentaban diferencias significativas $(P<0,05)$. Adicionalmente, se evaluó el crecimiento en distintas épocas del año para determinar en qué momento es más provechoso a nivel de crecimiento es uso de las mallas raschel, pudiendo especificar y recomendar tiempos de manejo en vivero.

\section{RESULTADOS}

Calidad e intensidad lumínica bajo mallas raschel. Solo las mallas raschel verde y azul fueron selectivas, modificando la calidad lumínica que entregan a las plantas, transmitiendo las longitudes de onda correspondiente a su color. Para el azul entre $450-550 \mathrm{~nm}$ y para el verde entre $500-550 \mathrm{~nm}$. Las mallas de color blanco y negro no modificaron su espectro de transmisión (figura 1).

A nivel de intensidad lumínica las mallas negra y azul aportan mayor sombra (70 y $60 \%$ de atenuación, respectivamente) que las mallas verde y blanco (55 y $50 \%$ ). Los porcentajes de sombra fueron calculados con respecto a la luz incidente durante un día despejado (figura 2).

Biomasa, altura y área foliar especifica. La biomasa total final de las plántulas de Pinus radiata y Eucalyptus globulus presentó diferencias significativas a nivel de tratamientos ( $P=0,016$ y $P=0,048$ respectivamente), no así para E. nitens. En todas las especies existieron diferencias significativas al comparar la biomasa generada con los dos tipos de contenedor, con valores más altos en plantas traspasadas a contenedor de $500 \mathrm{~cm}^{3}$ comparado con las mantenidas en contenedor de $130 \mathrm{~cm}^{3}$, que corresponden a las condiciones operacionales de vivero.

La relación vástago/raíz fue mayor en plántulas de ambas especies de Eucalyptus que en Pinus radiata. Exis-

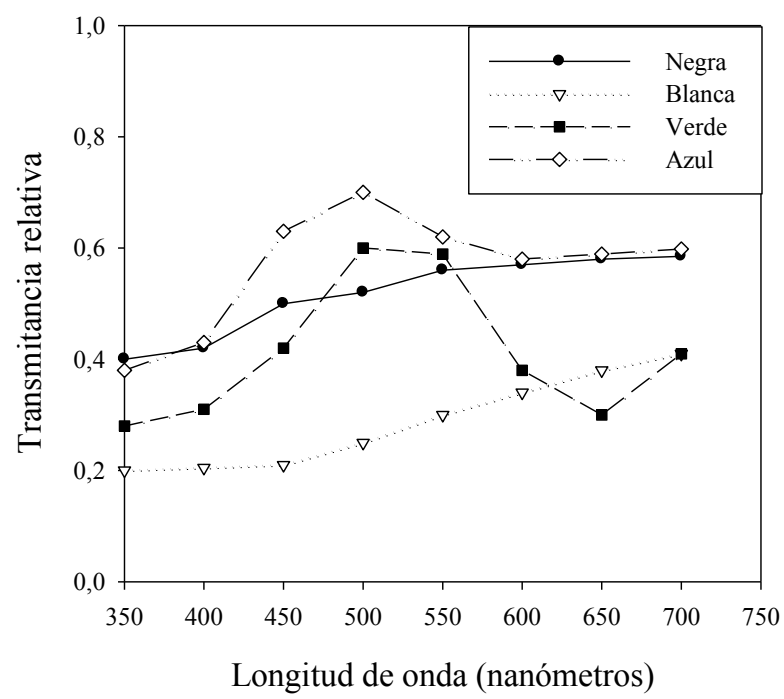

Figura 1. Calidad lumínica bajo mallas rashel de colores (negra, blanca, verde y azul). El espectro corresponde a la transmitancia relativa en un barrido de longitudes de onda desde los 350 a los $750 \mathrm{~nm}$ de cada malla de color medido en un espectrofotómetro UV-visible (ver metodología para detalles).

Light quality under raschel colored meshes $(\mathrm{N}=$ black, $\mathrm{A}=$ blue, $\mathrm{B}=$ white and $\mathrm{V}=$ green). The spectrum corresponds to relative transmittance on a range of wavelengths ranging from 350 to $750 \mathrm{~nm}$, using part of each mesh and spectrophotometrically measured the UVvisible light. (See methodology for details). 
tiendo diferencias significativas al comparar dicho valor en plantas de ambas especies de Eucalyptus cuando fueron transferidas a un contenedor de mayor volumen, donde aumenta significativamente en todos los tratamientos con mallas $(P<0,001)$. Si se comparan los tratamientos con mallas raschel de color; existen diferencias en $P$. radiata observándose el menor valor en el tratamiento sin malla, tanto en el contenedor de $130 \mathrm{~cm}^{3}$ como en el de $500 \mathrm{~cm}^{3}$; y los mayores valores bajo malla negra y azul (contenedor de $500 \mathrm{~cm}^{3}$ ), y bajo mallas negra, azul y verde (contenedor de $\left.130 \mathrm{~cm}^{3}\right)$. Eucalyptus nitens no presenta diferencias significativas entre tratamientos con mallas, en el valor de la relación vástago/raíz para ambos volúmenes de contenedor. Eucalyptus globulus solo presentó diferencias significativas en contenedor de $130 \mathrm{~cm}^{3}$ con malla azul, donde se registró el menor valor. Existe interacción significativa entre el factor mallas raschel de color con el aumento del tamaño del contenedor, en la relación vástago/raíz en ambas especies de Eucalyptus spp. $(P<0,04)$.

No existen diferencias significativas en la distribución de biomasa, entre hojas, tallo y raíz al usar mallas raschel de distinto color en especies de Eucalyptus spp. cuando se mantuvieron en contenedor de $500 \mathrm{~cm}^{3}$. Se observaron di-

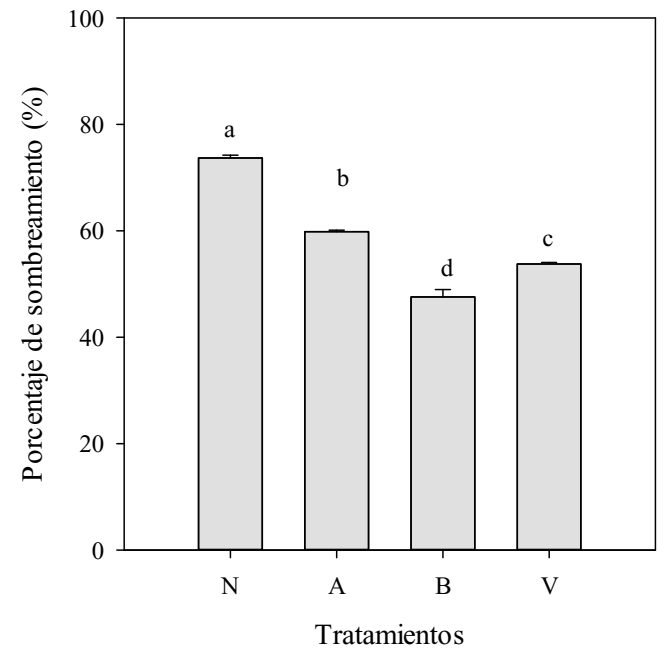

Figura 2. Intensidad lumínica bajo mallas rashel de colores ( $\mathrm{N}=$ negra, $\mathrm{A}=$ azul, $\mathrm{B}=$ blanca y $\mathrm{V}=$ verde). Porcentaje de sombreamiento o atenuación de la luz incidente total, producido por mallas raschel de colores utilizadas en el ensayo. Los promedios fueron calculados con cinco mediciones en tres momentos del día durante un día despejado usando un radiómetro. Los valores corresponden al promedio y su error estándar (ver detalles en metodología). Se indican diferencias significativas $(P<0,01)$ con letras distintas.

Light intensity under raschel colored meshes $(\mathrm{N}=$ black; $\mathrm{A}=$ blue; $\mathrm{B}=$ white and $\mathrm{V}=$ green). Shading percentage of full sunlight produced by the meshes that were used in the trial. The mean was calculated by using five measurements at three different times of a clear day using radiometer (Li-cor LI-250). The values correspond to mean and standard error (see methodology for details). Significant differences are showed with different letters $(P<0.01)$. ferencias significativas en la distribución de biomasa para E. globulus creciendo en contenedor de $130 \mathrm{~cm}^{3}$, con mayor proporción de raíz y menor proporción de biomasa en hojas, bajo malla azul (cuadro 2). En P. radiata se observaron diferencias significativas en la distribución de biomasa en distintos órganos, siendo menor la proporción de raíz cuando las plantas estás bajo malla raschel color negro y azul, para ambos volúmenes de contenedor. En tanto las plantas que se encuentran sin malla presentan una mayor proporción de biomasa hacia raíces y menor hacia hojas

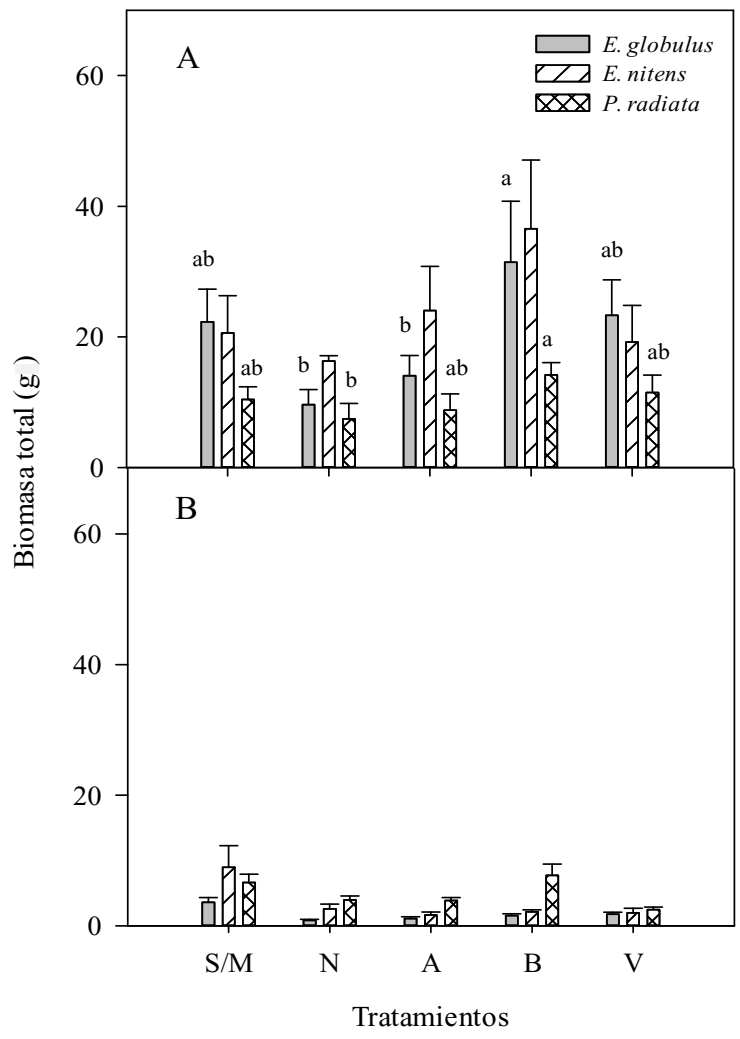

Figura 3. Biomasa total de plántulas de Eucalyptus globulus, Eucalyptus nitens y Pinus radiata mantenidas 1 año bajo mallas raschel de color: $\mathrm{N}=$ negra; $\mathrm{A}=$ azul; $\mathrm{B}=$ blanco; $\mathrm{V}=$ verde $\mathrm{y}$ un control $\mathrm{S} / \mathrm{M}=$ sin malla, plena luz. Se usaron dos tipos de contenedor: A $\left(500 \mathrm{~cm}^{3}\right)$ y B $\left(130 \mathrm{~cm}^{3}\right)$. Los valores corresponden al promedio de cuatro individuos para cada tratamiento para $E$. globulus, E. nitens y seis individuos para $P$. radiata con su error estándar. Letras minúsculas indican diferencias significativas entre tratamientos por especie $(P<0,001)$, no se incluyen letras cuando no hay diferencias entre tratamientos. No existe interacción significativa entre factores $(P>0,05)$.

Total biomass of seedlings of Eucalyptus globulus, E. nitens and Pinus radiata maintained for one year under colored mesh treatments.: $\mathrm{N}=$ black; $\mathrm{A}=$ blue; $\mathrm{B}=$ white; $\mathrm{V}=$ green and $\mathrm{S} / \mathrm{M}=$ without mesh, full sunlight. Two different volumes for the container were used: A $\left(500 \mathrm{~cm}^{3}\right)$ and $\mathrm{B}\left(130 \mathrm{~cm}^{3}\right)$. The value corresponds to the mean of four individuals per treatment for E. globulus, E. nitens and 6 individuals in $P$. radiata \pm the standard error. Lowercase letters showed significant differences among treatments of the same species $(P<0.001)$. There are not significant interaction between factors $(P>0.05)$. 
Cuadro 1. Relación vástago/raíz en plántulas de Eucalyptus globulus, Eucalyptus nitens y Pinus radiata después de nueve meses de exposición a diferentes mallas raschel de color.

Seedling shoot/root ratio for Eucalyptus globulus, E. nitens and Pinus radiata after 9 months grown below different colored meshes.

\begin{tabular}{lcccccc}
\hline \multicolumn{1}{c}{ Tratamientos } & E. globulus & E. nitens & P. radiata & E. globulus & E. nitens & P. radiata \\
\hline & \multicolumn{3}{c}{ Contenedor $500 \mathrm{~cm}^{3}$} & & & Contenedor $130 \mathrm{~cm}^{3}$ \\
\hline Sin malla & $7,0 \pm 1,4 \mathrm{a}$ & $6,3 \pm 1,3 \mathrm{a}$ & $2,8 \pm 0,2 \mathrm{~b}$ & $5,9 \pm 0,8 \mathrm{a}$ & $7,1 \pm 1,5 \mathrm{a}$ & $2,0 \pm 0,1 \mathrm{~b}$ \\
Negro & $8,2 \pm 0,8 \mathrm{a}$ & $9,5 \pm 1,1 \mathrm{a}$ & $3,9 \pm 0,3 \mathrm{a}$ & $5,3 \pm 0,2 \mathrm{a}$ & $7,5 \pm 1,3 \mathrm{a}$ & $3,7 \pm 0,2 \mathrm{a}$ \\
Azul & $7,6 \pm 0,5 \mathrm{a}$ & $9,8 \pm 0,9 \mathrm{a}$ & $3,9 \pm 0,4 \mathrm{a}$ & $2,3 \pm 0,1 \mathrm{~b}$ & $4,3 \pm 0,3 \mathrm{a}$ & $3,3 \pm 0,2 \mathrm{a}$ \\
Blanco & $5,9 \pm 1,0 \mathrm{a}$ & $8,8 \pm 0,6 \mathrm{a}$ & $3,1 \pm 0,3 \mathrm{ab}$ & $3,9 \pm 0,8 \mathrm{ab}$ & $4,0 \pm 0,2 \mathrm{a}$ & $2,8 \pm 0,4 \mathrm{ab}$ \\
Verde & $8,3 \pm 2,4 \mathrm{a}$ & $6,4 \pm 1,2 \mathrm{a}$ & $3,5 \pm 0,2 \mathrm{ab}$ & $4,0 \pm 0,5 \mathrm{a}$ & $4,4 \pm 1,4 \mathrm{a}$ & $5,3 \pm 0,9 \mathrm{a}$ \\
\hline
\end{tabular}

Nota: Los tratamientos corresponden a: Sin malla, negro, azul, blanco y verde. Se usaron dos tipos de contenedor: $500 \mathrm{~cm}^{3}$ y $130 \mathrm{~cm}^{3}$. Cada valor corresponde al promedio de 4 muestras de plántulas en ambas especies de Eucalyptus y 6 plántulas en $P$. radiata con el error estándar. Las letras minúsculas indican diferencias significativas entre tratamientos por especie $(P<0,01)$. Existe interacción estadísticamente significativa para Eucalyptus spp. $(P<0,05)$.

The raschel colored mesh treatments correspond: $\mathrm{N}=$ black; $\mathrm{A}=$ blue; $\mathrm{B}=$ white; $\mathrm{V}=$ green and $\mathrm{S} / \mathrm{M}=$ without mesh, full sunlight. The volume of containers that were used: $\mathrm{A}\left(500 \mathrm{~cm}^{3}\right)$ and $\mathrm{B}\left(130 \mathrm{~cm}^{3}\right)$. Each value represents the mean of four independent samples for each treatment for E. globulus, $E$. nitens and six independent samples for $P$. radiata with standard error. Lowercase letters showed significant differences among treatments at the same species $(P<0.01)$.

Cuadro 2. Proporción de biomasa relativa seca en plántulas de Eucalyptus globulus, Eucalyptus nitens y Pinus radiata (\%) bajo tratamientos con mallas raschel de colores. Los tratamientos corresponden a: Control sin malla $\mathrm{S} / \mathrm{M}, \mathrm{N}=$ negro, $\mathrm{A}=\mathrm{azul}, \mathrm{B}=\mathrm{blanco} \mathrm{y}$ $\mathrm{V}=$ verde. Se usaron dos tipos de contenedor: $500 \mathrm{~cm}^{3}$ y $130 \mathrm{~cm}^{3}$. Cada valor corresponde al promedio de cuatro plántulas en ambas especies de Eucalyptus y seis plántulas en P. radiata con el error estándar. Letras minúsculas indican diferencias significativas entre tratamientos para una misma especie según parte de la planta ${ }^{*}$ indican diferencias entre tipos de contenedor.

Proportion of dry biomass (\%) in seedlings of Eucalyptus globulus, E. nitens and Pinus radiata grown under different raschel mesh colors. Treatments for the colored raschel meshes are: $\mathrm{N}=$ black, $\mathrm{A}=$ blue, $\mathrm{B}=$ white, $\mathrm{V}=$ green and $\mathrm{S} / \mathrm{M}=$ without mesh. Additionally, two types of containers were used: $500 \mathrm{~cm}^{3}$ and $130 \mathrm{~cm}^{3}$. Each value is the average of four seedlings in both species of Eucalyptus and six seedlings in P. radiata with standard error. Lowercase letters indicate significant differences among treatments for the same kind of structure and * indicates differences among container types.

\begin{tabular}{|c|c|c|c|c|c|c|c|c|c|}
\hline \multicolumn{4}{|c|}{ Eucalyptus globulus } & \multicolumn{3}{|c|}{ Eucalyptus nitens } & \multicolumn{3}{|c|}{ Pinus radiata } \\
\hline \multicolumn{10}{|c|}{ Contenedor de $500 \mathrm{~cm}^{3}$} \\
\hline Tts & Hojas & Tallo & Raíz & Hojas & Tallo & Raíz & Hojas & Tallo & Raíz \\
\hline $\mathrm{S} / \mathrm{M}$ & $44 \pm 1,1 \mathrm{a}$ & $42 \pm 2,2 \mathrm{a}$ & $14 \pm 2,84 \mathrm{a}$ & $46 \pm 5,2 \mathrm{a}$ & $38 \pm 1,6 \mathrm{a}$ & $16 \pm 4,1 \mathrm{a}$ & $48 \pm 1,9 \mathrm{a}$ & $25 \pm 1,1 \mathrm{a}$ & $27 \pm 1,0 \mathrm{a}$ \\
\hline $\mathrm{N}$ & $43 \pm 2,5 \mathrm{a}$ & $46 \pm 2,8 \mathrm{a}$ & $11 \pm 0,97 \mathrm{a}$ & $43 \pm 1,9 \mathrm{a}$ & $48 \pm 1,0 \mathrm{a}^{*}$ & $10 \pm 1,0 \mathrm{a}$ & $54 \pm 2,2 \mathrm{a}$ & $26 \pm 1,8 \mathrm{a}$ & $21 \pm 0,8 \mathrm{~b}$ \\
\hline A & $43 \pm 5,9 \mathrm{a}$ & $45 \pm 6,5 \mathrm{a}$ & $12 \pm 0,71 \mathrm{a}^{*}$ & $51 \pm 2,0 \mathrm{a}$ & $39 \pm 2,2 \mathrm{a}$ & $9 \pm 0,9 \mathrm{a}^{*}$ & $52 \pm 2,8 \mathrm{a}$ & $27 \pm 1,9 \mathrm{a}$ & $21 \pm 1,4 \mathrm{~b}$ \\
\hline $\mathrm{B}$ & $35 \pm 0,4 \mathrm{a}$ & $49 \pm 2,9 \mathrm{a}$ & $16 \pm 2,9 \mathrm{a}^{*}$ & $46 \pm 1,7 \mathrm{a}$ & $44 \pm 1,2 \mathrm{a}$ & $10 \pm 0,6 a^{*}$ & $50 \pm 2,2 \mathrm{a}$ & $25 \pm 1,7 \mathrm{a}$ & $25 \pm 1,6 \mathrm{ab}$ \\
\hline $\mathrm{V}$ & $38 \pm 1,2 \mathrm{a}$ & $49 \pm 2,9 \mathrm{a}$ & $13 \pm 2,8 \mathrm{a}$ & $46 \pm 2,6 \mathrm{a}$ & $39 \pm 1,5 \mathrm{a}$ & $15 \pm 2,3 \mathrm{a}$ & $53 \pm 1,8 \mathrm{a}$ & $25 \pm 1,5 \mathrm{a}$ & $23 \pm 0,9 a b$ \\
\hline \multicolumn{10}{|c|}{ Contenedor de $130 \mathrm{~cm}^{3}$} \\
\hline Tts & Hojas & Tallo & Raíz & Hojas & Tallo & Raíz & Hojas & Tallo & Raíz \\
\hline $\mathrm{S} / \mathrm{M}$ & $47 \pm 2,0 \mathrm{a}$ & $38 \pm 1,4 \mathrm{a}$ & $15 \pm 1,9 b$ & $53 \pm 1,7 \mathrm{a}$ & $34 \pm 1,1 \mathrm{a}$ & $13 \pm 2,0 \mathrm{a}$ & $43 \pm 1,5 b$ & $23 \pm 1,6 \mathrm{a}$ & $34 \pm 1,0 \mathrm{a}$ \\
\hline $\mathrm{N}$ & $37 \pm 2,7 \mathrm{ab}$ & $47 \pm 2,5 \mathrm{a}$ & $16 \pm 0,6 b$ & $48 \pm 4,2 \mathrm{a}$ & $39 \pm 1,8 \mathrm{a}^{*}$ & $13 \pm 2,5 \mathrm{a}$ & $54 \pm 2,0 \mathrm{a}$ & $24 \pm 2,5 \mathrm{a}$ & $21 \pm 1,0 \mathrm{c}$ \\
\hline $\mathrm{A}$ & $29 \pm 2,2 b$ & $40 \pm 1,5 \mathrm{a}$ & $31 \pm 0,9 \mathrm{a}^{*}$ & $40 \pm 1,7 \mathrm{a}$ & $41 \pm 2,2 \mathrm{a}$ & $19 \pm 0,9 a^{*}$ & $48 \pm 1,6 b$ & $29 \pm 1,4 \mathrm{a}$ & $23 \pm 1,2 c$ \\
\hline B & $38 \pm 1,8 \mathrm{ab}$ & $41 \pm 1,2 \mathrm{a}$ & $22 \pm 2,6 b *$ & $44 \pm 1,4 \mathrm{a}$ & $35 \pm 1,0 \mathrm{a}$ & $20 \pm 0,8 a^{*}$ & $45 \pm 4,6 \mathrm{ab}$ & $28 \pm 2,5 \mathrm{a}$ & $28 \pm 2,3 \mathrm{bc}$ \\
\hline $\mathrm{V}$ & $44 \pm 2,3 \mathrm{a}$ & $43 \pm 1,8 \mathrm{a}$ & $13 \pm 0,6 \mathrm{c}$ & $45 \pm 3,2 \mathrm{a}$ & $34 \pm 8,1 \mathrm{a}$ & $21 \pm 6,3 \mathrm{a}$ & $47 \pm 1,5 \mathrm{ab}$ & $23 \pm 1,9 \mathrm{a}$ & $29 \pm 1,5 b$ \\
\hline
\end{tabular}


en contendedor de $130 \mathrm{~cm}^{3}$. En E. nitens se registraron diferencias en la distribución de tallo y raíz al comparar plantas mantenidas en ambos contenedores, aumentando la proporción de tallo (malla negra) y disminuyendo el de raíces (azul y blanca) en contenedor de mayor volumen (cuadro 2). En P. radiata en tanto no existen diferencias en la distribución de biomasa entre tamaños de contenedor. Solo existe interacción significativa entre el efecto de las mallas raschel y el tamaño del contenedor en E. globulus y $E$. nitens, no en $P$. radiata (análisis estadísticos en anexo).

El área foliar específica, SLA $\left(\mathrm{cm}^{2} \mathrm{~g}^{-1}\right)$ presenta diferencias significativas en E. globulus cuando las plantas desarrollan sus hojas bajo distintas mallas raschel de color, para ambos contenedores (figura 4). El valor de SLA

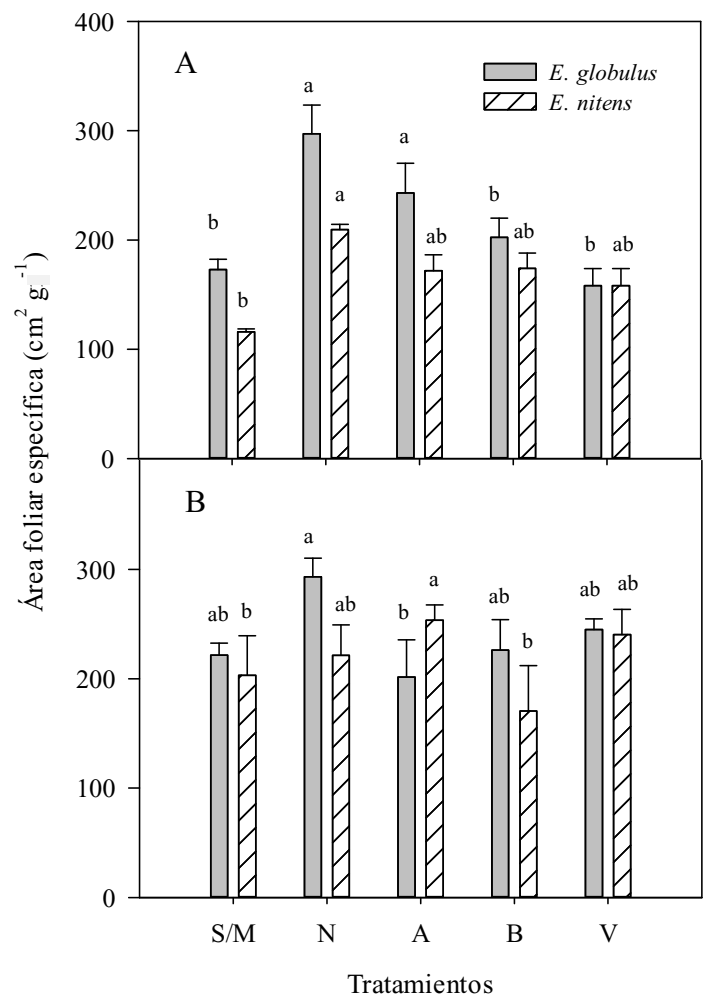

Figura 4. Área foliar específica, $\left(\mathrm{cm}^{2} \mathrm{~g}^{-1}\right)$ en plántulas de Eucalyptus globulus y E. nitens bajo mallas raschel de distintos colores: $\mathrm{N}=$ negro, $\mathrm{A}=$ azul, $\mathrm{B}=$ blanco y $\mathrm{V}=$ verde $\mathrm{y}$ un control $\mathrm{S} / \mathrm{M}=$ sin malla en dos volúmenes de contenedor: A $\left(500 \mathrm{~cm}^{3}\right)$ y B $\left(130 \mathrm{~cm}^{3}\right)$. Los valores corresponden al promedio de cuatro plántulas por especie y por tratamiento \pm error estándar. Letras minúsculas indican diferencias significativas entre tratamientos por especie $(P<0,001)$. No existe interacción entre ambos factores $(P>0,05)$.

The specific leaf area (SLA: $\mathrm{cm}^{2} / \mathrm{g}$ ) of seedlings of Eucalyptus globulus and E. nitens under colored mesh treatments: $\mathrm{N}=$ black; $\mathrm{A}=$ blue; $\mathrm{B}=$ white; $\mathrm{V}=$ green and $\mathrm{S} / \mathrm{M}=$ without mesh, full sunlight grown in two different volume containers: $\mathrm{A}\left(500 \mathrm{~cm}^{3}\right)$ and $\mathrm{B}\left(130 \mathrm{~cm}^{3}\right)$. The value corresponds to the mean of four seedlings per species and treatment \pm standard error. Lowercase letters showed significant differences among treatments per species $(P<0.001)$. There are not significant interaction between factors $(P>0.05)$. para E. globulus es mayor cuando se encuentra bajo malla negra y azul (contenedor de $500 \mathrm{~cm}^{3}$ ) y en malla negra (contenedor de $130 \mathrm{~cm}^{3}$ ), es decir, presentan hojas más delgadas y expandidas. E. nitens presenta mayor SLA bajo malla negra comparado con el valor de SLA de hojas desarrolladas sin malla (contenedor $500 \mathrm{~cm}^{3}$ ) y en malla azul, comparado con tratamiento sin malla o blanca (contenedor $\left.130 \mathrm{~cm}^{3}\right)$. No existe interacción entre volumen de contenedor y color de malla raschel para ninguna de las dos especies (análisis estadísticos en anexo).

El incremento relativo en altura total presentó diferencias significativas entre mallas raschel de colores en $E$. nitens y $P$. radiata $(P=0,048$ y $P=0,007$, respectivamente), siendo mayor el incremento cuando las plantas crecieron bajo malla raschel color blanco, comparado con tratamiento sin malla en $E$. nitens y bajo malla blanca comparado con negra y azul, en el caso de $P$. radiata (figura 5). El valor más alto de incremento relativo se encontró en $E$. nitens con malla blanca ( $300 \%$ ) y el más bajo en $P$. radiata con malla azul y negra $(90 \%)$. No existe interacción entre tratamientos de mallas raschel de color y especies $(P>0,05)$.

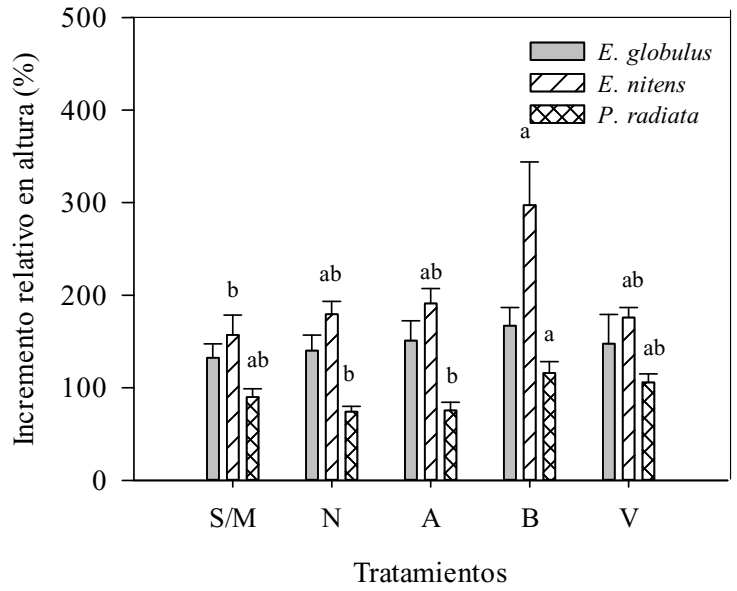

Figura 5. Incremento relativo en altura durante nueve meses (marzo a noviembre) en plántulas de Eucalyptus globulus, Eucalyptus nitens y Pinus radiata bajo mallas raschel de distintos colores: negra (N), azul (A), blanca (B) y verde (V) y un control $\mathrm{S} / \mathrm{M}=$ sin malla en contenedor de $130 \mathrm{~cm}^{3}$. Cada valor corresponde a un promedio de 14 individuos en $P$. radiata y 10 en E. globulus y E. nitens \pm error estándar. Letras minúsculas indican diferencias significativas entre tratamientos por especie $(P<0,001)$. No existe interacción entre los tratamientos de mallas raschel y especies $(P>0,05)$, para detalles ver Materiales y Métodos.

Relative increment in height (\%) for nine months (MarchNovember) in Eucalyptus globulus, E. nitens and Pinus radiata grown under raschel meshes of different colors: $\mathrm{N}=$ black; $\mathrm{A}=$ blue; $\mathrm{B}=$ white; $\mathrm{V}=$ green and $\mathrm{S} / \mathrm{M}=$ without mesh, full sunlight in a $130 \mathrm{~cm}^{3}$ container. Each value corresponds to the mean of 14 individuals in $P$. radiata and 10 individuals of E. globulus and E. nitens \pm the standard error. Lowercase letters indicate significant differences among treatments per species $(P<0.001)$. There are not significant interaction between treatments and species $(P>0.05)$, for details see Materials and Methods. 
Al comparar el incremento relativo en altura, según intervalos de tiempo bajo mallas raschel de color, se presentaron diferencias significativas $(P<0,001)$ en $P$. radiata y E. nitens, no así en E. globulus para ningún intervalo definido de tiempo (figura 6). En E. nitens las diferencias significativas de crecimiento relativo entre tratamientos de mallas de colores, se encontraron en los periodos de marzo-mayo y julio-septiembre. En $P$. radiata las diferencias significativas entre tratamiento con mallas se encontraron solo en el periodo julio-septiembre. Con respecto a la estacionalidad del crecimiento, $P$. radiata presentó sus mayores incrementos de altura durante marzo - mayo (otoño) y septiembre - noviembre (primavera), sin embargo, no mostró diferencias al estar bajo distintas mallas raschel ( $P=0,293$ y $P=0,103$, respectivamente). En tanto, E. globulus y E. nitens mostraron su mayor crecimiento en el periodo septiembre-noviembre (primavera), no existiendo diferencias significativas entre tratamientos (E. globulus $P=0,486 ; E$. nitens $P=0,103$ ) (figura 6). No existe interacción entre tratamientos de mallas raschel de colores y los periodos de crecimiento $(P>0.05)$ para ninguna especie (análisis estadísticos en anexo).

Eficiencia fotoquímica máxima. La fluorescencia máxima potencial del PSII evaluado con el parámetro $(\mathrm{Fv} / \mathrm{Fm})$ indica la vitalidad de las plantas. Las dos especies de $\mathrm{Eu}$ calyptus no presentaron diferencias significativas entre mallas raschel de colores durante invierno y primavera, siendo sus valores cercanos al óptimo de $0,83(P>0,05)$, evidenciando que el uso de las mallas no daña la vitalidad de las plantas. En tanto, P. radiata presentó valores significativamente más bajos en primavera $(0,75)$ en el tratamiento sin malla comparado con la malla negra y azul $(P<0,001)$ (figura 7).

\section{DISCUSIÓN}

Aunque las mallas de colores han sido usadas ampliamente en viveros forestales, este trabajo aporta los primeros resultados que miden la calidad lumínica de dichas mallas. La sombra que realmente aportan las mallas fue cercana al que indican los proveedores. Con respecto a la calidad de luz, se determinó que solo la malla azul y verde, son efectivamente selectivas (figura 1), ya que modifican el espectro de luz que reciben las plantas. En tanto, las mallas negra y blanca solo aportan sombra en distintas proporciones, sin modificar la calidad lumínica.

En general, la malla blanca promovió las mejores respuestas de crecimiento en biomasa y altura, siendo la más recomendable para los viveros forestales que deseen mejorar el crecimiento. Aunque en este trabajo no se evaluó la tasa fotosintética de las plantas, el mayor crecimiento observado se podría explicar porque las plantas bajo malla blanca se encuentran posiblemente cerca de su óptimo lumínico, donde podrían presentar su mayor tasa de fotosíntesis. Determinaciones de la tasa fotosintética en respuesta

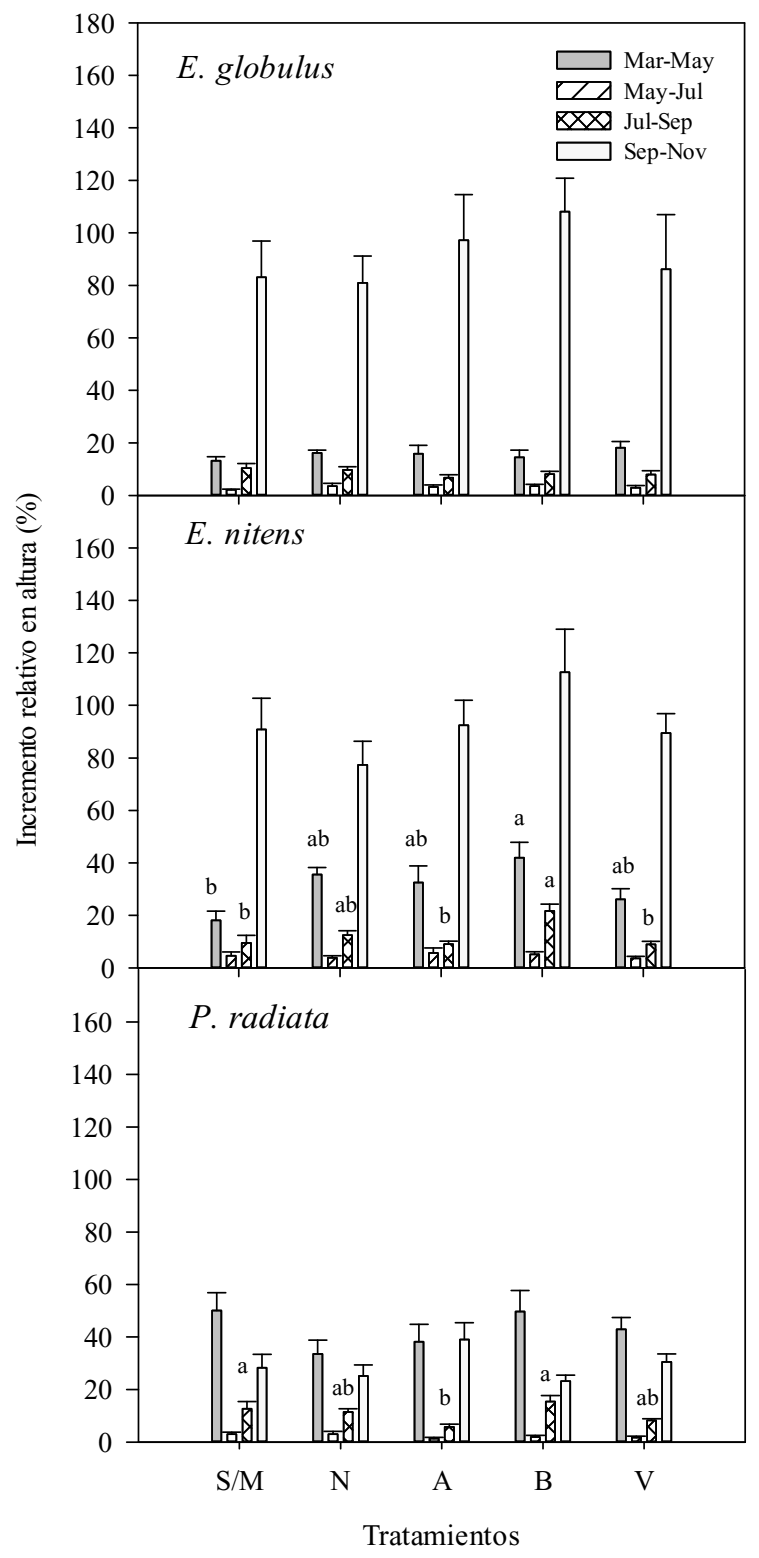

Figura 6. Incremento relativo en altura (\%) evaluados en distintos periodos del año: a) marzo-mayo, b) mayo-julio, c) julioseptiembre y d) septiembre-noviembre en plántulas de Eucalyptus globulus, Eucalyptus nitens y Pinus radiata, bajo mallas raschel de distintos colores: $\mathrm{S} / \mathrm{M}=$ sin malla; $\mathrm{N}=$ negra, $\mathrm{A}=$ azul, $\mathrm{B}=$ blanca y $\mathrm{V}=$ verde, en contenedor de $130 \mathrm{~cm}^{3}$. Cada valor corresponde a un promedio de 14 individuos en $P$. radiata y 10 individuos en E. globulus y E. nitens \pm error estándar. Letras minúsculas indican diferencias significativas entre tratamientos por especie $(P<$ $0,001)$. Ausencia de letras cuando no hay diferencias significativas entre tratamientos. No existe interacción entre factores $(P>0,05)$.

Relative increment in height (\%) during different periods of the year: A) March-May, B) May-July, C) July-September and D) SeptemberNovember on Eucalyptus globulus, E. nitens and Pinus radiata seedlings grown under raschel meshes of different colors: $\mathrm{N}=$ black; $\mathrm{A}=$ blue; $\mathrm{B}=$ white; $\mathrm{V}=$ green and $\mathrm{S} / \mathrm{M}=$ without mesh, full sunlight in a $130 \mathrm{~cm}^{3}$ container. Each value corresponds to the mean of 14 individuals in $P$. radiata and 10 in E. globules and E. nitens \pm the standard error. Lowercase letters indicate significant differences among treatments per species $(P<0.001)$. There are not significant interaction between factors $(P>0.05)$. 


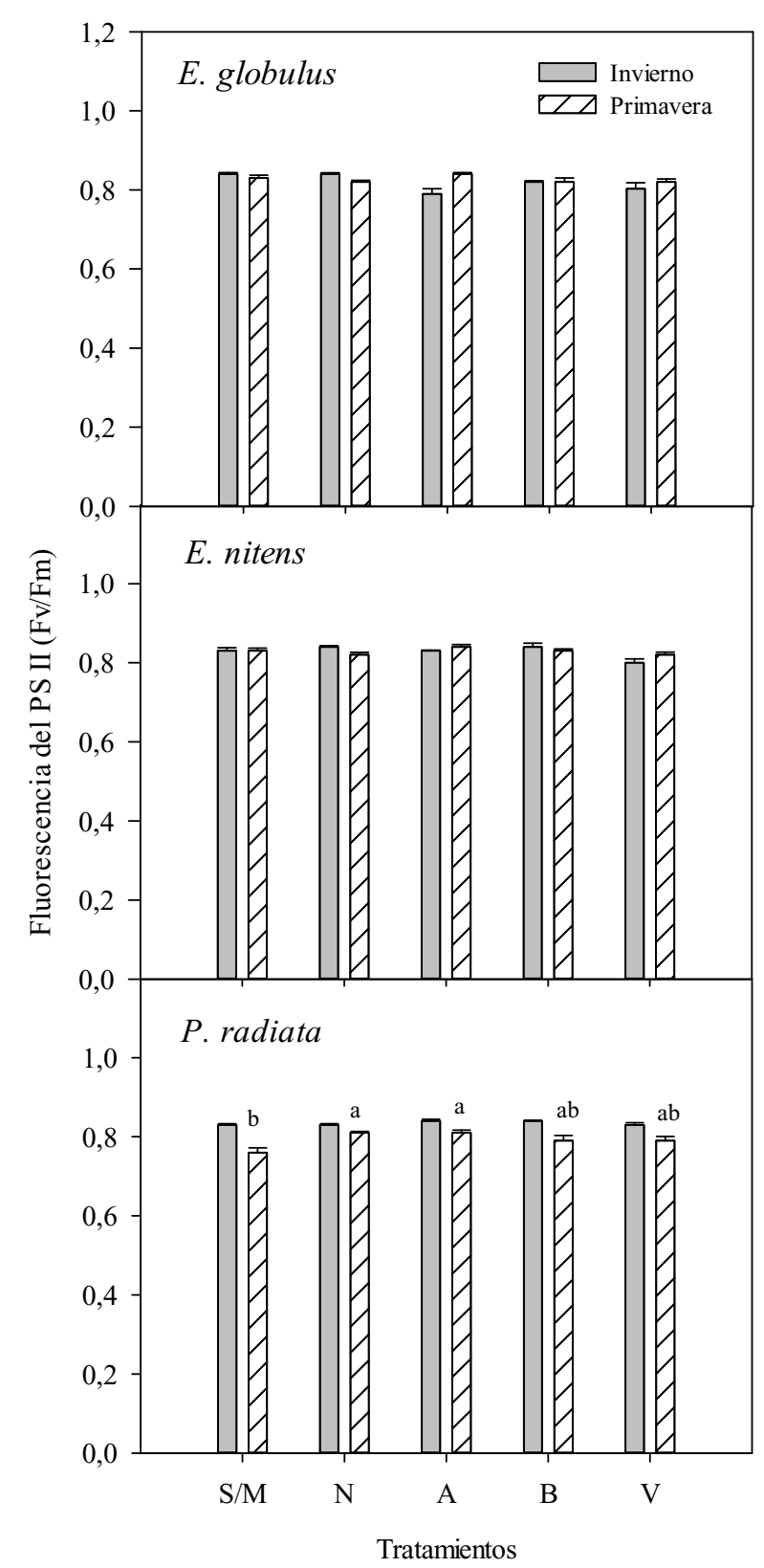

Figura 7. Eficiencia fotoquímica máxima $(\mathrm{Fv} / \mathrm{Fm})$, indicador de vitalidad en plántulas de Eucalyptus globulus, Eucalyptus nitens y Pinus radiata bajo mallas raschel de distintos colores: $\mathrm{N}=$ negra, $\mathrm{A}=$ azul, $\mathrm{B}=$ blanca y $\mathrm{V}=$ verde y un control, $\mathrm{S} / \mathrm{M}$ $=$ sin malla en contenedor de $130 \mathrm{~cm}^{3}$. Las plántulas fueron evaluadas en invierno y primavera. Cada valor corresponde a un promedio de seis individuos \pm error estándar. Letras minúsculas indican diferencias significativas entre tratamientos por especie $(P<0,01)$.

Maximum quantum efficiency of PSII photochemistry (Fv/ Fm), used as vitality indicator for Eucalyptus globulus, E. nitens and Pinus radiata seedlings grown under raschel meshes of different colors: $\mathrm{N}=$ black; $\mathrm{A}=$ blue; $\mathrm{B}=$ white; $\mathrm{V}=$ green and $\mathrm{S} / \mathrm{M}=$ without mesh, full sunlight in a $130 \mathrm{~cm}^{3}$ container. Seedlings were evaluated in winter and spring. Each value corresponds to the mean of six individuals \pm the standard error. Lowercase letters showed significant differences among treatments per species $(P<0.01)$. a luz, apoyan esta explicación y revelan que a $800 \mu \mathrm{mol}$ de fotones $\mathrm{m}^{-2} \mathrm{~s}^{-1}$ se obtiene el máximo valor de fotosíntesis para plántulas de E. globulus en la zona de Valdivia (datos no publicados, Bustos-Salazar A.). Considerando que la máxima intensidad lumínica en un día despejado (1.500 a $2.000 \mu \mathrm{mol}$ de fotones $\left.\mathrm{m}^{-2} \mathrm{~s}^{-1}\right)$, la intensidad lumínica bajo malla blanca posiblemente presente valores cercanos al óptimo, ya que estas estructuras producen una atenuación del $50 \%$ de la luz incidente. Por otra parte, posiblemente las mallas que proporcionan mayor sombra (negra y azul), podrían llegar a limitar la fotosíntesis y el crecimiento, provocando también un desbalance en la asignación de biomasa, favoreciendo sólo el desarrollo de la parte aérea (Lambers et al. 2008), siendo esto negativo para la sobrevivencia de plantas que serían trasladadas a plantación desde el vivero.

Otro factor capaz de provocar modificaciones en el balance de biomasa de las plantas es el aumento del volumen de contenedor (Landis 1990, Landis et al. 2009). Las plantas de Eucalyptus nitens y Eucalytus globulus son sensibles al aumento del volumen de contenedor, produciendo plantas más desbalanceadas (10:1). Al contrario, $P$. radiata fue más insensible al aumento del volumen de contenedor en este experimento. Según Haase (2008) los valores reportados para la relación parte aérea/raíz en especies de vivero en una planta ideal a raíz desnuda, es del orden de 3:1 y en plantas producidas en contenedor la relación es de $2: 1$. El crecimiento radical es un parámetro muy relevante para la sobrevivencia de los individuos al ser llevados a plantación, por ende, deben tener un sistema radical homogéneo (Haase 2008).

El efecto de la modificación del ambiente lumínico en plantas ha sido poco estudiado en especies forestales. Sin embargo, existe información en algunas especies herbáceas mediante el uso de filtros de colores, por ejemplo, el filtro color rojo promueve plantas con mayor número de brotes en Cyperus rotundus L. (Lazo y Ascencio 2010). Otra especie de herbácea en la cual se aplicó filtros selectivos de color fue Arabidopsis thaliana L. Heynh, donde se destaca que el uso de filtro color verde promueve una mayor elongación de entrenudos (Folta 2004). En nuestro trabajo, en cambio se encontró que en general la malla blanca, que aporta una sombra intermedia, generó un mayor incremento en altura y plantas de mayor biomasa final.

Estudios con mallas raschel de color, también han sido realizados en hortalizas, helechos, plantas ornamentales y frutales (Oren-Shamir et al. 2001, Rodríguez et al. 2015). Los resultados muestran una amplia variación; desde mayor crecimiento en ramas y hojas con malla roja, hasta menor crecimiento y ramas más cortas con malla azul (Oren-Shamir et al. 2001). En nuestro experimento, contrariamente a lo esperado se observó diferencias significativas en la biomasa total solo en $P$. radiata y E. globulus, al comparar distintas mallas raschel y cuando estas plantas crecieron en contenedor de $500 \mathrm{~cm}^{3}$. Solo en la malla negra se redujo significativamente el crecimiento en 
P. radiata, y en mallas negras y azul en E. globulus. Esto probablemente fue producto de la limitación fotosintética impuesta por estas mallas que generan mayor sombra. En especies vegetales hortícolas como Capsicum anuиm L. se ha reportado un mayor rendimiento bajo malla color blanco, tanto en el peso total y número de frutos (ÁlvarezAvilés et al. 2010), atribuible a una mayor disponibilidad de carbohidratos provenientes de la fotosíntesis. Existen pocos antecedentes del efecto de estas mallas sobre el crecimiento de especies forestales en vivero, sin embargo, para Nothofagus alessandri Espinosa se ha observado un mayor crecimiento cuando las plántulas son cultivadas con un $35 \%$ de sombra (Santelices et al. 2011), cercana al nivel de sombra generada por la malla blanca, en este trabajo la cual coincidentemente promovió un mayor crecimiento en $P$. radiata y E. globulus.

La vitalidad de las plantas evaluada a través del desempeño fotoquímico a nivel del fotosistema II ( $\mathrm{Fv} / \mathrm{Fm}$ ), no presentó diferencias significativas, ni efectos negativos en dichos tratamientos. Sin embargo, fue significativamente menor en plántulas de $P$. radiata $(0,75)$ que se encontraron sin malla raschel (figura 7). Este resultado puede ser indicativo de cierto grado de ajuste del aparato fotosintético a variaciones en la temperatura primaveral en una condición de plena luz. Los valores reportados para las otras especies fueron siempre cercanos al óptimo $(0,83)$, indicando la máxima vitalidad bajo estas condiciones. Este parámetro $(\mathrm{Fv} / \mathrm{Fm})$ ha sido usado ampliamente para determinar vitalidad en plantas (Baker 2008, Bustos-Salazar 2011), y su uso como parámetros de fluorescencia a nivel de fotosistema II podría ser una herramienta de fácil aplicación y no destructiva para detectar posibles daños en plantas a nivel de vivero. Esto podría constituir una fuente de información importante para la toma de decisiones, y la posible aplicación de nuevos tratamientos a nivel forestal.

En los viveros forestales es usual que se sombree en forma estacional (verano) con mallas raschel para proteger las plántulas durante sus estados iniciales, en el presente experimento se trabajó con plantas de tres meses de edad al inicio, las que fueron mantenidas durante todo el año con mallas raschel. Desde el punto de vista fisiológico y considerando el crecimiento, se puede destacar que la aplicación de una sombra moderada con malla blanca mejora el desempeño de las plantas, aún aplicada durante un año completo. Al evaluar el crecimiento en distintos periodos y bajo mallas raschel de color, pudimos identificar los momentos de mayor crecimiento. Basado en estos resultados podríamos proponer que se utilizara malla de color blanco para obtener un mayor crecimiento y posteriormente retirarlas para endurecer las plantas previas a la plantación. Para llevar a la práctica este procedimiento también es necesario considerar la estacionalidad del crecimiento de cada especie y el momento de plantación. La variación observada en el crecimiento en Pinus radiata, era esperable considerando los resultados descritos para individuos adultos de esta especie en la zona edafoclimática de suelos trumaos en Chile mostrando dos puntos de máximo crecimiento; a principios de verano y mediados de otoño (Toral et al. 2005), lo cual coincide con los pulsos que nosotros observamos en nuestro experimento.

Como era esperable, las hojas de las dos especies de Eucalyptus fueron más delgadas (mayor SLA) cuando las plantas crecieron bajo malla azul y negra, esto ha sido ampliamente reportado en especies de latifoliadas (Terashima et al. 2005). Una mayor área foliar específica también se puede considerar negativa al momento de llevar a plantación, ya que hojas más delgadas y expandidas tienen una mayor superficie transpiratoria (Steubing et al. 2002), esto puede ser especialmente crítico en ambientes con alta demanda evaporativa. También hojas más delgadas pueden ser más susceptibles a tener daño por heladas. En ciertos viveros es frecuente que se corte una parte de las hojas para evitar excesiva transpiración como es el caso de radal (Lomatia hirsuta Lam.), ulmo (Eucryphia cordifolia Cav.) y tiaca (Caldcluvia paniculata (Cav.) D. Don) (Escobar y Donoso 2011). En todo caso las prácticas de vivero deben orientarse de acuerdo con el tipo de planta que se quiere producir y el uso que se le quiera dar, i.e. plantación con fines productivos, restauración de ecosistemas forestales, protección de riberas de río, así también debe considerarse los extremos ambientales que puedan darse en cada sitio de plantación.

\section{CONCLUSIONES}

Las mallas raschel de colores presentan diferencias significativas a nivel de intensidad (porcentaje de sombra) y calidad lumínica (longitud de onda) los que influyen a nivel morfológico y fisiológico en las tres especies estudiadas. Las mallas de color negro y azul promueven el mayor nivel de sombra en un rango similar al informado por los proveedores y solo las mallas de color verde y azul transmiten selectivamente.

Considerando el crecimiento en altura y biomasa, así como un mayor grosor de las hojas en Eucalyptus spp. se propone el uso de malla de color blanca, aplicada en forma estacional diferida, considerando los máximos de crecimiento para cada especie. En cambio, si se desea obtener plantas más balanceadas (mayor desarrollo radical) se recomienda usar malla azul.

\section{AGRADECIMIENTOS}

Al Dr. Daniel Calderini, de la Facultad de Ciencias Agrarias y al Dr. Ignacio Moreno del Laboratorio de Polímeros de la Facultad de Ciencias, ambos de la Universidad Austral de Chile, por el préstamo de equipamiento. Al Sr. Jaime Büchner (viverista), a la Sra. Cecilia Muñoz por facilitar el material vegetal y a Forestal Valdivia S.A. A. Bustos-Salazar agradece a Laboratorio de Fisiología Vegetal, Facultad de Ciencias, CONICYT N² 21140737 (beca de doctorado nacional) y CONICYT/FONDAP 15110009. 


\section{REFERENCIAS}

Álvarez- Avilés A, FA Romo Ayala, P Valenzuela Cornejo, MA Huez López, EJ López El, F Preciado Flores, D Sandoval Fernández. 2010. Efecto de las mallas sombreadoras de color sobre el rendimiento y calidad del chile jalapeño (Capsicum annum L.) 'Tajin', en la costa de Hermosillo. In XXXV Congreso Nacional de la Ciencia del Suelo "El suelo, sustento de vida y nuestro mejor aliado contra el cambio climático". 25 al 29 de octubre de 2010, Mexicali Baja California, México.

Bustos-Salazar A. 2011. Efecto de la intensidad lumínica en el crecimiento de plántulas de Embothrium coccineum en procedencias contrastantes una aproximación morfológica, fisiológica y genética. Tesis Magíster en Ciencias Vegetales. Valdivia, Chile. Facultad de Ciencias Agrarias. Universidad Austral de Chile. 89 p.

Baker NR. 2008. Chlorophyll Fluorescence: A Probe of Photosynthesis In Vivo. Annual Review of Plant Biology 59: 89-113. DOI: $\underline{59.032607 .092759}$

Escobar B, G Donoso. 2011. Técnicas de propagación de especies nativas. Taller de producción de plantas. Santiago, Chile. Ministerio del Medio Ambiente. 26 p.

Folta KM. 2004. Green light stimulates early stem elongation antagonizing light-mediated growth inhibition. Plant Physiology 135: 1407-1416. DOI: $10.1104 / p p .104 .038893$

Haase DL. 2008. Understanding Forest Seedling Quality: Measurements and Interpretation. Tree Planters' Notes 52 (2): 24-30.

INFOR (Instituto Forestal, CL). 2015. Anuario Forestal 2015. Santiago, Chile. INFOR. 174 p. (Boletín Estadístico $\mathrm{N}^{\circ}$ 150).

Lambers H, FS Chapin, TL Pons. 2008. Plant Physiological Ecology. New York, USA. Springer. 604 p.

Landis TD. Containers: types and functions. 1990. In Landis TD, RK Dumroese, DL Haase eds. The container tree nursery manual. Agriculture Handbook 674. Washington DC, U.S.A. Department of Agriculture, Forest Service. p. 1-40.

Landis TD. The Target Plant Concept. 2. 2009. In Dumroese RK, L Tara, TD Landis eds. Nursery manual for native plants: A guide for tribal nurseries 1: Nursery management. Agriculture Handbook 730.Washington DC, U.S.A. Department of Agriculture, Forest Service. p. 15-31.

Lazo J, J Ascencio. 2010. Efecto de diferentes calidades de luz sobre el crecimiento de Cyperus rotundus. Bioagro 22 (2): 153-158.

Lorrain S, T Allen, PD Duek, GC Whitelam, Ch Fankhauser. 2008. Phytochrome-mediated inhibition of shade avoi- dance involves degradation of growth-promoting bHLH transcription factors. The Plant Journal 53: 312-323. DOI: 10.1111/j.1365-313X.2007.03341. x.

Luebert F, P Pliscoff. 2006. Sinopsis bioclimática y vegetacional de Chile. Santiago, Chile. Editorial Universitaria. 316 p.

Myers JA, K Kitajima. 2007. Carbohydrate storage enhances seedling shade and stress tolerance in a neotropical forest. Journal of Ecology 95: 383-395. DOI: 10.1111/j.13652745.2006. 01207.x

Oren-Shamir M, E Gussakovsky, E Eliezer, A Nissim-Levi, K Ratner, R Ovadia, Y Giller, Y Shahak. 2001. Coloured shade nets can improve the yield and quality of green decorative branches of Pittosporum variegatum. The Journal of Horticultural Science and Biotechnology 76(3): 353-361. DOI: https://doi.org/10.1080/14620316.2001.11511377

Quiroz I, B Gutiérrez. 2014. Propuesta Reglamento Para Semillas y Plantas Forestales. Concepción, Chile. INFOR-SAGINNOVA-CORFO. 74 p.

Reich PB, MB Walters, DS Ellsworth. 1997. From tropics to tundra: Global convergence in plant functioning. Proceeding of National Academy of Sciences of the United States of America 94: 13730-13734. DOI: 10.1073/pnas.94.25.13730

Rodríguez MM, DM Morales. 2015. Efecto de mallas sombreadoras sobre la producción y calidad de

frutos de arándano (Vaccinium corymbosum L.) cv. Brigitta. Scientia Agropecuaria 6(1): 41-50. DOI: 10.17268/ sci.agropecu.2015.01.04.

Santelices R, RM Navarro Cerrillos, F Drake, C Mena. 2011. Efecto de la cobertura y de la fertilización en el desarrollo de plantas de Nothofagus alessandrii cultivadas en contenedor. Bosque 32(1): 85-88. DOI: 10.4067/S071792002011000100010 .

Schlatter J, R Grez, V Gerding. 2003. Manual para el reconocimiento de suelos. $3^{\mathrm{a}}$ ed. Valdivia, Chile. Universidad Austral de Chile. 114 p.

Steubing L, R Godoy, M Alberdi. 2002. Métodos de ecología vegetal. Santiago, Chile. Editorial Universitaria. 345.

Terashima I, YT Handa, Y Tazoe, P Vyas, S Yano. 2005. Irradiance and phenotype: comparative eco- development of sun and shade leaves in relation to photosynthetic $\mathrm{CO}_{2}$ diffusion. Journal of Experimental Botany: Phenothypic Plasticity and the Changing Environment Special Issue. 57(2): 343-354. DOI: 10.1093/jxb/erj014.

Toral M, A Fratti, L González. 2005. Crecimiento estacional y rentabilidad de plantaciones forestales comerciales de pino radiata en suelos de trumao según método de establecimiento. Bosque 26(1): 43-54. DOI: https://doi.org/10.4067/ $\underline{\mathrm{s} 0717-92002005000100005}$ 
Anexo. Análisis estadísticos.

Appendix. Stadistical analysis.

\begin{tabular}{|c|c|c|c|c|c|c|c|c|}
\hline \multirow[b]{2}{*}{ Variable } & \multirow[b]{2}{*}{ FV } & \multirow[b]{2}{*}{$\mathrm{DF}$} & \multicolumn{2}{|c|}{ E. globulus } & \multicolumn{2}{|c|}{ E. nitens } & \multicolumn{2}{|c|}{ P. radiata } \\
\hline & & & $\mathrm{F}$ & $P$ & $\mathrm{~F}$ & $P$ & $\mathrm{~F}$ & $P$ \\
\hline \multirow[t]{3}{*}{ Tallo/Raíz } & Contenedor & 1 & 21,136 & $<0,001$ & 14,66 & $<0,001$ & 14,338 & $<0,001$ \\
\hline & Mallas & 4 & 4,531 & 0,006 & 1,872 & 0,141 & 10,611 & $<0,001$ \\
\hline & Interacción & 4 & 4,264 & 0,008 & 2,892 & 0,039 & 1,064 & 0,384 \\
\hline \multirow[t]{3}{*}{ Biomasa total } & Contenedor & 1 & 1,511 & 0,228 & 40,234 & $<0,001$ & 23,941 & $<0,001$ \\
\hline & Mallas & 4 & 0,921 & 0,465 & 1,189 & 0,336 & 3,362 & 0,016 \\
\hline & Interacción & 4 & 0,523 & 0,719 & 1,863 & 0,143 & 0,361 & 0,835 \\
\hline \multirow[t]{3}{*}{ Proporción de biomasa hojas } & Contenedor & 1 & 1,255 & 0,271 & 0,036 & 0,85 & 6,634 & 0,013 \\
\hline & Mallas & 4 & 4,326 & 0,007 & 0,795 & 0,538 & 3,668 & 0,011 \\
\hline & Interacción & 4 & 4,827 & 0,004 & 3,541 & 0,018 & 0,559 & 0,693 \\
\hline \multirow[t]{3}{*}{ Proporción de biomasa tallo } & Contenedor & 1 & 5,389 & 0,027 & 6,978 & 0,013 & 0,109 & 0,917 \\
\hline & Mallas & 4 & 1,608 & 0,198 & 1,9 & 0,136 & 1,71 & 0,163 \\
\hline & Interacción & 4 & 0,657 & 0,627 & 1,09 & 0,379 & 0,728 & 0,577 \\
\hline \multirow[t]{3}{*}{ Proporción de biomasa raíz } & Contenedor & 1 & 25,074 & $<0,001$ & 9,175 & 0,005 & 20,891 & $<0,001$ \\
\hline & Mallas & 4 & 6,858 & $<0,001$ & 1,359 & 0,271 & 13,692 & $<0,001$ \\
\hline & Interacción & 4 & 7,571 & $<0,001$ & 1,703 & 0,175 & 2,059 & 0,1 \\
\hline \multirow[t]{3}{*}{ Área foliar específica (SLA) } & Contenedor & 1 & 0,146 & 0,705 & 10,822 & 0,003 & - & - \\
\hline & Mallas & 4 & 6,945 & $<0,001$ & 6,587 & $<0,001$ & - & - \\
\hline & Interacción & 4 & 1,237 & 0,316 & 1,29 & 0,296 & - & - \\
\hline \multirow[t]{3}{*}{ IR altura periodos } & Mallas & 4 & 0,505 & 0,732 & 5,522 & $<0,001$ & 2,533 & 0,041 \\
\hline & Periodos & 3 & 0,378 & 0,769 & 276,265 & $<0,001$ & 0,418 & 0,74 \\
\hline & Interacción & 12 & 0,491 & 0,918 & 1,239 & 0,259 & 0,696 & 0,755 \\
\hline IR altura total & Mallas & 4 & 0,364 & 0,833 & H: 9,592 & 0,048 & 3,921 & 0,007 \\
\hline \multirow[t]{3}{*}{$\mathrm{Fv} / \mathrm{Fm}$} & Mallas & 4 & 2,238 & 0,072 & 2,107 & 0,088 & 4,116 & 0,004 \\
\hline & Periodos & 1 & 0,01 & 0,92 & 1,753 & 0,189 & 19,348 & $<0,001$ \\
\hline & Interacción & 4 & 1,412 & 0,238 & 0,889 & 0,474 & 3,1 & 0,02 \\
\hline
\end{tabular}

IR altura: se realizó una ANOVA de dos vías con los factores (mallas y especies), para evaluar si existe interacción, sin embargo, no hubo interacción $(P=0,419)$. 